\title{
ANTERIOR CERVICAL DISCECTOMY WITH OR WITHOUT FUSION IN NON-TRAUMATIC SINGLE LEVEL CERVICAL DISC DISORDER: STUDY OF 50 CASES
}

\author{
DAS $\mathrm{S}^{1}$, RASHID MM ${ }^{2}$, ZAHANKFI $^{3}, \mathrm{KHAN} \mathrm{SI}^{4}$
}

\begin{abstract}
Objectives: The aim of this study is to compare patients undergoing single level anterior cervical discectomy without fusion (ACD) versus anterior cervical discectomy with fusion (ACDF).

Methods: A retrospective analysis of 50 patients with degenerative cervical spondylosis of them 25 had undergone ACD without fusion and remaining 25 undergone ACDF at either C4-C5, C5C6 level or at C6-C7 level.

Results: The kinematic analysis included the range of motion, intervertebral angulations, anteroposterior translation and disc height assessed for the cervical functional spinal units at the operated level and adjacent levels. At the operated level of C4-C5, C5-C6 and C6-C7, the range of motion and the translation were minimal in the anterior cervical discectomy without fusion ( $A C D$ ) group, but absent in the cervical discectomy with fusion (ACDF) group. The superior adjacent levels range of motion and the translation were greater in the ACDF group compared with the ACD group. But both groups had almost similar results in term of hospital stay, mean time for improvement and patient satisfaction.

Conclusion: The clinical results of anterior cervical discectomy without fusion (ACD) and anterior cervical discectomy with fusion ( $A C D F$ ) were comparable. In cervical discectomy without fusion, the elastic fibrous intradiscal scar at the operated level allows a small degree of mobility and the adjacent cervical levels are not overstressed. Fusion is not routinely required in single level cervical disc herniation until it is associated with instability, loss of cervical lordosis, hard disc, osteophytic bar and multi-segmental disease. So ACD is a better option in single level cervical disc disorder than ACDF.
\end{abstract}

Keywords: Anterior cervical discectomy without fusion, Anterior cervical discectomy with fusion, Elastic fibrous intradiscal scar.

J Dhaka Med Coll. 2018; 27(1) : 29-35

\section{Introduction}

Cervical disc herniation is a common pathology of the cervical spine and the surgical treatment can be Anterior Cervical Discectomy without fusion (ACD), Anterior Cervical Discectomy and Spinal Fusion (ACDF), posterior cervical discectomy, anterior or posterior foraminotomy, percutaneous cervical nucleoplasty or cervical artificial disc replacement. Anterior Cervical Discectomy without fusion (ACD) and Anterior Cervical Discectomy with Fusion (ACDF) are common approaches among spine surgeons for most cervical herniated discs ${ }^{1,2}$.

The anterior cervical decompression with fusion with a bone graft harvested from the iliac crest began to be used during the years 1955-1959 and since 1975 anterior discectomy without fusion for cervical disc herniation was introduced. Many variations of the cervical anterior approaches have been presented over the last six decades such as: discectomy;

1. Dr. Sukriti Das, Associate Professor, Dept of Neurosurgery, Dhaka Medical College Hospital.

2. Dr. Md. Mamunur Rashid, Resident, Phase B, Dept of Neurosurgery, Dhaka Medical College Hospital

3. Dr. Kanij Fatema Ishrat Zahan, Assistant Professor, Dept of Neurosurgery, Dhaka Medical College Hospital

4. Dr. Samsul Islam Khan, Medical Officer, Dept of Neurosurgery, Dhaka Medical College Hospital.

Correspondence: Dr. Sukriti Das, Associate Professor, Department of Neurosurgery, Dhaka Medical College \& Hospital, Dhaka. Cell Phone: +8801711676848, Email: sukriti66@yahoo.com.

Received: 21 July 2017 Revision: 01 September 2017

Accepted: 15 September 2017 
discectomy and fusion (autologous or homologous graft; intersomatic spacer-metallic, biological polymers; bone inductors; with plates) $)^{2-4}$.

These surgical treatments lead to the decompression of compressed neural elements and the stability of the cervical spine, without abnormal movements. Most spine surgeons consider that the anterior cervical discectomy without fusion must be limited to one space rather than multiple spaces. Also it is believed that the anterior cervical discectomy with fusion may lead to acceleration of degenerative changes at the immediately adjacent discal levels secondary to abnormal spinal motion ${ }^{5-}$ 7. The cervical intervertebral disc should not be entirely removed in anterior cervical discectomy without fusion and the cartilaginous endplates should be left intact, but the entire cervical disc should be removed and the vertebral body endplates must be decorticated in anterior cervical discectomy with fusion. Most spine surgeons consider that the results of these two types of approaches
(ACD and ACDF) are comparable both in terms of the decompression and the cervical stability $^{8-10}$. The healing after the discectomy without fusion leads to an intradiscal fibrous scar and not a real bone fusion because the cartilaginous endplates should be left intact in ACD.

This study tried to compare the differences of anterior cervical discectomy without fusion (ACD) versus anterior cervical discectomy with fusion $(\mathrm{ACDF})$ in this study.

\section{Material and Methods}

This study performed a comparative study of patients undergoing one level anterior cervical discectomy without fusion versus anterior cervical discectomy with fusion. The study included fifty patients operated at either C4-C5, C5-C6 level or at the C6-C7 level: a group of anterior cervical discectomy without fusion performed at one level on 25 patients was matched to a second group of 25 patients with single-level of anterior cervical discectomy with fusion, based on level, age and sex.

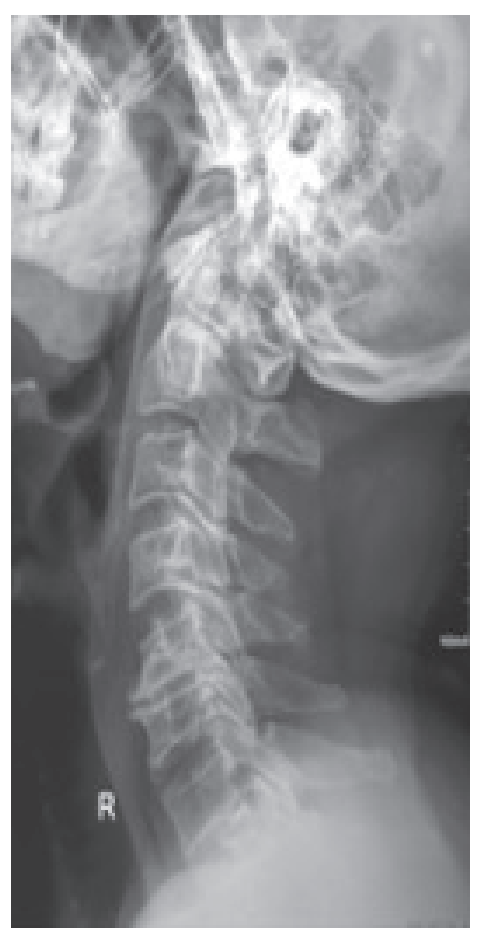

Figure: Pre-op radiograph of cervical spondylosis@C5-C6

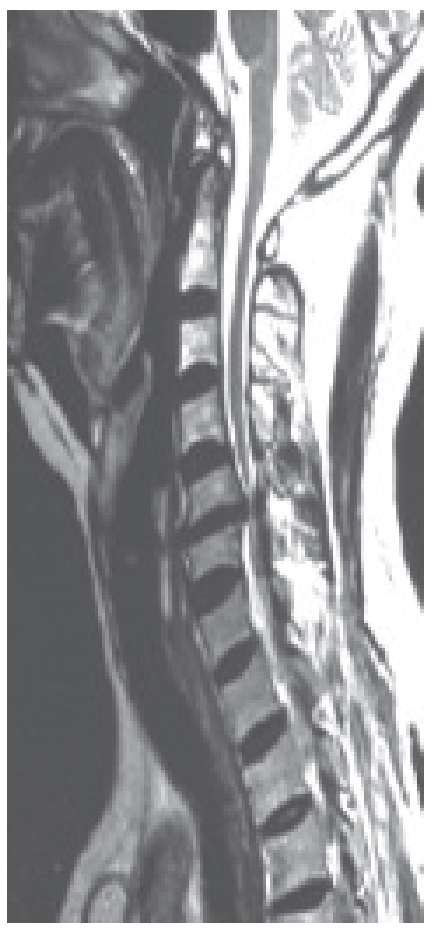

Figure: Pre-op MRI of cervical spondylosis@C5-C6

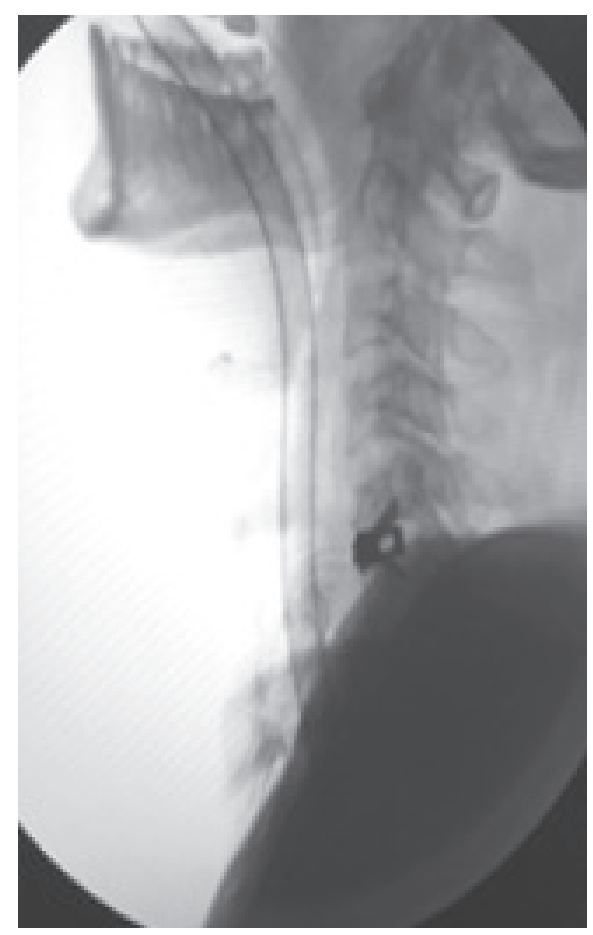

Figure: Post-op radiograph after ACDF@,C5-C6 
Inclusion criteria for the ACD and ACDF groups: all patients included for the ACD and ACDF groups presented with clinical symptoms due to one level herniated cervical disc of degenerative origin; all patients had preoperative complete general and neurological examination, they had preoperative MRI and plain radiographs of the cervical spine anterioposterior, lateral neutral, flexion and extension obtained preoperatively. As exclusion criteria: the patients with clinical or imaging evidence (MRI, X-rays) of additional diseased cervical spine, history of cervical spinal injury were excluded from the study. Also the patients with cervical instability at the level of disc herniation, which need a possible fusion and fixation, were excluded from the group of ACD patients.

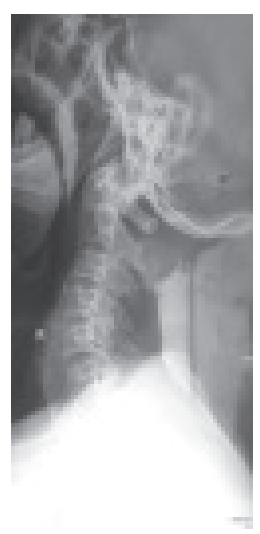

Fig.: Pre-op radiograph of cervical disc herniation@ C4-C5

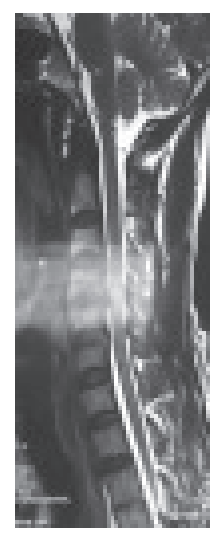

Fig.: Pre-op MRI of cervical disc herniation@ C4-C5

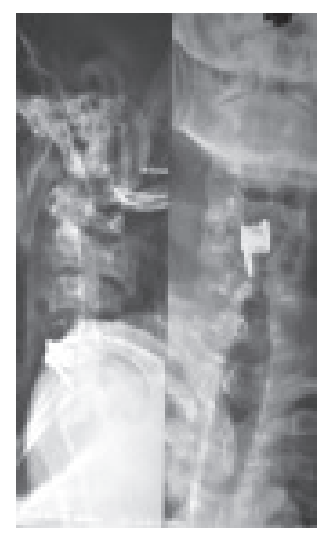

Fig.: Post-op radiograph after ACDF@C4-C5

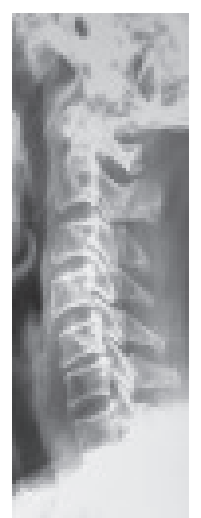

Fig.: Pre-op radio-graph of cervical disc herniation (a) C5-C6

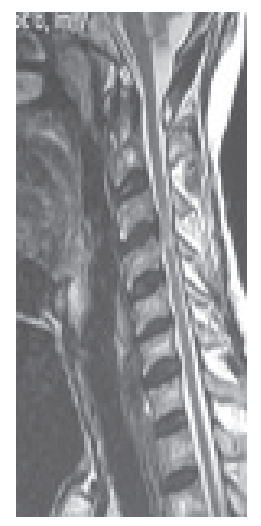

Fig.: Pre-op MRI of cervical disc herniation@ C5-C6

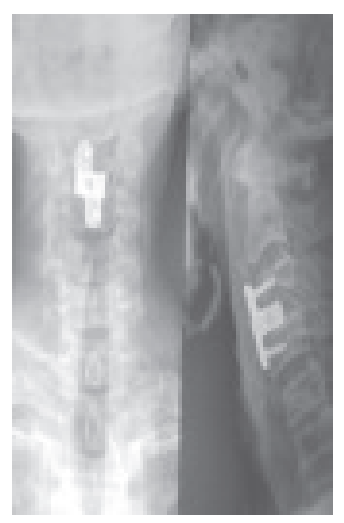

Fig.: Post-op radiograph after ACDF@C5-C6

These patients had presented with cervical radiculopathy, myelopathy or radiculomyelopathy and the diagnosis was confirmed on imaging. Decision regarding procedure to be performed (ACD, ACDF) was made preoperatively in most of the cases. ACD was performed in patients who presented with radiculopathy or myelopathy due to disc disease with normal lordosis and without any obvious instability or subluxations. Fusions were performed in patients having hard discs, osteophytic bars, instability and loss of cervical lordosis.

Each patient's age, sex, occupation, duration of symptoms, signs, radiological findings, details of operative procedure performed, postoperative hospital stay, and postoperative complications, postoperatively intervertebral translations during cervical flexion and extension were recorded. Patients were followed up after three months and one year. The success of the fusion was assessed by plain lateral radiograph of the cervical spine performed at follow up. 


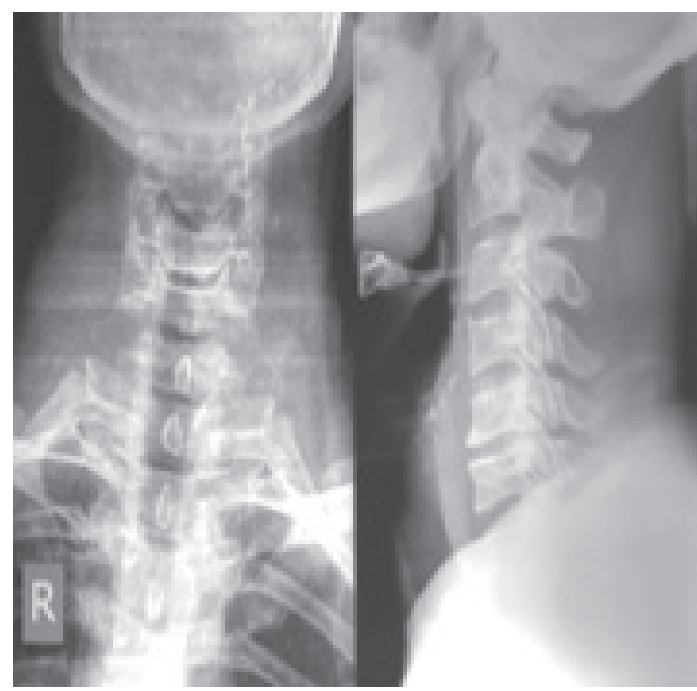

Figure: Pre-op radiograph of cervical disc herniation@C4-C5

\section{Results}

All the 50 patients operated by anterior approach were available for follow up for one year or more. There were 25 patients in ACD group and 25 patients in ACDF. Demographic and clinical data of the patients are summarized in Table I The patients in the ACD group were younger than $\mathrm{ACDF}$ group. Male preponderance was seen in both groups.

\section{Table-I}

Patients data

\begin{tabular}{llcc}
\hline & ACD & ACDF & \\
\hline Sex & Male & 20 & 21 \\
Age & Female & 5 & 4 \\
Symptoms & Mean (yr) & 39.4 & 43.4 \\
& Range (yr) & $17-65$ & $30-71$ \\
& Mean duration & 3.25 & 4.91 \\
& (months) & & \\
& Neck, arm pain & $21 / 25$ & $22 / 25$ \\
& Numbness & $24 / 25$ & $24 / 25$ \\
Presentation & Weakness & $10 / 25$ & $15 / 25$ \\
& Radiculopathy & $17 / 25$ & $7 / 25$ \\
& Myelopathy & $5 / 25$ & $14 / 25$ \\
& Radiculo- & $3 / 25$ & $4 / 25$ \\
& myelopathy & & \\
\hline
\end{tabular}

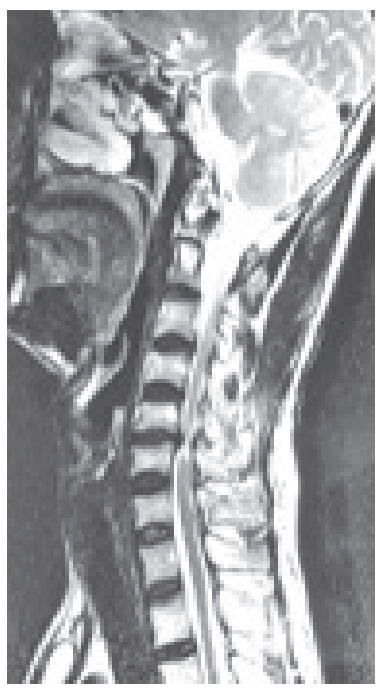

Figure: Pre-op MRI of cervical disc herniation (a) C4-C5

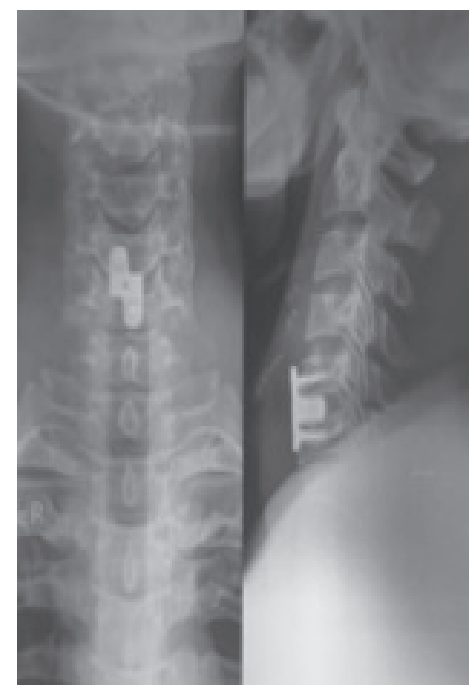

Figure: Post-op radiograph afterACDF@C4-C5
Majority of patients with radiculopathy (17 patients) underwent $A C D$, but it was also done in patients with milder degree of myelopathy $(5$ patients) and radiculomyelopathy (3 patients). Involved disc level was C4-C5, C5-C6 and C6C7 most common C5-C6.

As summarized in Table-II, ACD group had 2 complications, out of which 1 were procedure related (hoarseness of voice). In ACDF group had 9 complications, out of which 5 were procedure related ( 2 were Hoarseness of voice, 1 was post-operative weakness, 2 were dysphagia), 3 were systemic complication (2 were Pneumonia and 1 was neck ache with occipital headache), 1 patient had persistent graft site pain.

Table-II

Complications associated with procedure

\begin{tabular}{llcc}
\hline Complication & ACD & ACDF \\
\hline Local & Hoarseness of voice & 1 & 2 \\
& Post-operative & Nil & 1 \\
& weakness & & \\
& Dysphagia & Nil & 2 \\
Systemic & Pneumonia & 1 & 2 \\
& Neckache with & Nil & 1 \\
& occipital headache & & \\
Graft site & Infection & Nil & 1 \\
\hline
\end{tabular}


Lowest mean post-op hospital stay was for ACD group (5.1 days) and ACDF group (6.3 days). The difference in mean post-op hospital stay between ACD and ACDF group was not statistically significant.

Table -III shows data for follow-up. In ACD group there was more rapid improvement (5.5 days) as compared to ACDF group (6.7 days). In terms of mean time for improvement to start, there was statistically significant difference between ACD and ACDF group. Mean time to return to maximum normal status was 2.5 month for ACD group and 3.64 month for ACDF group. This was statistically not significant between ACD and ACDF group.

At the operated level the range of motion and the translation were present in the ACD group, for operated level, C5-C6 and C6-C7 and were absent in the ACDF group.

\section{Table-III}

\section{Follow-up}

\begin{tabular}{lcc}
\hline & ACD & ACDF \\
\hline $\begin{array}{l}\text { Mean time of post-op } \\
\text { hospital stay (days) }\end{array}$ & 5.1 & 6.3 \\
$\begin{array}{l}\text { Mean time to start } \\
\text { improvement (days) }\end{array}$ & 5.5 & 6.7 \\
$\begin{array}{l}\text { Mean time to return max. } \\
\text { normal status (month) }\end{array}$ & 2.5 & 3.64 \\
Patients satisfaction & & \\
- Excellent & 5 & 4 \\
- Good & 17 & 16 \\
- Fair & 2 & 3 \\
- Poor & 1 & 1 \\
- Graft site pain & 0 & 1 \\
\hline
\end{tabular}

\section{Discussion}

Anterior Cervical discectomy without fusion (ACD) and Anterior Cervical Discectomy with Fusion (ACDF) are the most commonly used approaches in cervical disc herniation. Most spine surgeons consider that the clinical results of these two types of approaches are comparable $8,10,12$. Some spine surgeons consider that the anterior cervical discectomy without fusion is limited to one space. But most surgeons consider that the anterior cervical discectomy with fusion may lead to acceleration of degenerative changes at immediately adjacent discal levels secondary to abnormal spinal motion $^{12-14}$.

In this study, patients of both radiculopathy and myelopathy were included as compared to similar other studies in which patient with only radiculopathy were included. In this retrospective study, random allocation of the patients was not done and decision regarding the procedure to be performed was made using certain criteria earlier mentioned. In this study we determined the differences outcome after anterior cervical discectomy without fusion versus anterior cervical discectomy with fusion in two groups.

ACD group experienced fewer complications (2 complications in 25 patients) as compared to ACDF group (9 complications in 25 patients). Hoarseness of voice was seen in one patient of ACD group. This was thought to be due to the traction on the recurrent laryngeal nerve. This complication is well described in literature and the incidence ranges from 1.1 to $2.4 \% .14,{ }^{19}$

Short term follow up showed that mean time for improvement was similar in ACD and ACDF group. Also ACD group returned to their maximum normal status much earlier than ACDF group. At the time of return to work, ACD and ACDF group had almost similar results in terms of patient's satisfaction. The low patient satisfaction in ACDF group can be accounted by the fact that majority patients in that group had myelopathy. As in patients of cervical myelopathy surgical results were modest, good initial results were expected in only about $70 \%$ of patients and functional outcome noticeably declined with long term follow up. ${ }^{20}$ Although outcome in patients with myelopathy was not as good as patients with radiculopathy, it seemed to be a result of natural history of the lesion and not of the operative technique. ${ }^{21}$

The comparison of our results with the normal data showed the translation was present in the ACD group at both operated levels and the translation was absent in the ACDF group at the operated level. The absence of intervertebral translation at the operated level is explained by intervertebral fusion in the ACDF group. The presence of lower values of intervertebral translation at the operated level in the ACD 
group is normal and is not a spinal instability. The healing at the operated level after the ACD consists of an intradiscal fibrous scar and not a real bone fusion because the cartilaginous endplates should be left intact. This elastic fibrous intradiscal scar ensures the cervical stability and allows a small degree of mobility at the operated level in the ACD group.

In the ACD group the means of the intervertebral translation were close to normal values at superjacent levels and in the ACDF group the intervertebral translation was greater compared with the normal values and with the ACD group. Also the comparison of superjacent translations found that the $\mathrm{C} 6-\mathrm{C} 7$ level fusion produced a higher increase in the $\mathrm{C} 3-\mathrm{C} 4$ level translation than the C5-C6 level fusion, therefore the range of motion is higher at a more distant level where the amplitude of movement may be higher. These results may explain why ACDF may lead to acceleration of degenerative changes at immediately overstressed superjacent discal levels secondary to abnormal spinal motion.

In this study the clinical results of these two types of anterior cervical approaches were comparable and this result is consistent with the literature. Nandoe et al. presented the long term outcome of a large series of patients after anterior cervical discectomy without fusion and compared that to results published on the longterm outcome after ACDF. Their results showed that ACD surgery is comparable to the results of ACDF. Also they concluded "because the superiority of any fusion procedure has never been proven, it has been suggested that fusion might not be necessary at all" ${ }^{15}$.

Botelho et al. studied the effectiveness of ACD compared with ACDF and concluded that the clinical results of $A C D$ and $A C D F$ are not significantly different, the addition of the intervertebral cage can enhance clinical results and the anterior cervical plate does not change the clinical results of ACD. Also they noted that $A C D$ produces lower rate of fusion than ACDF, but in ACD there is not a real fusion, there is an elastic fibrous intradiscal scar that ensures a very good cervical stability at the operated level ${ }^{16}$.
Kim et al. analysed the whole spine sagittal alignment after ACDF and concluded that the fusion procedure affects the spine sagittal alignment: cervical lordosis decreased especially in patients with high cervical lordosis and the sagittal vertical axis decreased [17].

The results of this study and the review of the literature showed that ACD has better clinical results than ACDF in single level cervical disc herniation and ACD ensures postoperatively a very good symptomatic improvement spinal alignment, motion and neural decompression.

\section{Conclusions}

The clinical results of anterior cervical discectomy without fusion and anterior cervical discectomy with fusion were comparable. In anterior cervical discectomy without fusion the elastic fibrous intradiscal scar at the operated level allows a small degree of mobility and the adjacent cervical levels are not overstressed. Anterior cervical discectomy with fusion may lead to acceleration of degenerative changes at immediately overstressed adjacent discal levels secondary to greater intervertebral translation at these adjacent levels.

No need for anterior cervical discectomy with fusion to trait a single level cervical disc herniation than in selected cases with preoperative instability at same level. Anterior cervical discectomy without fusion is a valid option in patients with one level cervical disc herniation without local instability.

Considering symptomatic improvement, less complication, easy and rapid to perform surgery, maintaining spinal motion and alignment and finally no need of any costly synthetic device, till today ACD is better option than ACDF.

\section{References}

1. Sugawara T. Anterior cervical spine surgery for degenerative disease: A review.

2. Neurol Med Chir 2015; 55: 540-546.

3. Gebremariam L, Koes BW, Peul WC, Huisstede BM. Evaluation of treatment effectiveness for the herniated cervical disc: A systematic review. Spine 2012; 37:E109-118.

4. Caruso R, Pesce A, Marrocco L, Wierzbicki V. Anterior approach to the cervical spine for treatment of spondylosis or disc herniation: Long-term results. 
Comparison between ACD, ACDF, TDR. Clin Ter 2014; 165: e263-270.

5. Song KJ, Choi BY. Current concepts of anterior cervical discectomy and fusion: A review of literature. Asian Spine J 2014; 8: 531-539.

6. Yang J, Hai Y, Pang C, Li H, Zu D, Zhu G, Xia X, Pei B. Biomechanical study on the effect of the length of cervical anterior fusion on adjacent levels. Zhonghua Wai Ke Za Zhi 2014; 52: 692-696.

7. Xia LZ, Zheng YP, Xu HG, Liu P. Effect of anterior cervical discectomy and fusion on adjacent segments in rabbits. Int J Clin Exp Med 2014; 7: 4291-4299.

8. Oktenoglu T, Cosar M, Ozer AF, Iplikcioglu C, Sasani M, Canbulat N,Sarioglu AC. Anterior cervical microdiscectomy with or without fusion. J Spinal Disord Tech 2007; 20: 361-368.

9. van Eck CF, Regan C, Donaldson WF, Kang JD, Lee JY. The revision rate and occurrence of adjacent segment disease after anterior cervical discectomy and fusion: a study of 672 consecutive patients. Spine 2014; 39:2143-2147.

10. Quintana LM. Complications in anterior cervical discectomy and fusion for cervical degenerative disc disease. World Neurosurg 2014; 82: 1058-1059.

11. Rosenthal P, Kim KD. Cervical adjacent segment pathology following fusion: Is it due to fusion? World J Orthop 2013;4: 112-113.

12. Wu SK, Kuo LC, Lan HC, Tsai SW, Chen CL, Su FC. The quantitative measurements of the intervertebral angulation and translation during cervical flexion and extension. Eur Spine J 2007; 16: 1435-1444
13. Lundine KM, Davis G, Rogers M, Staples M, Quan G. Prevalence of adjacent segment disc degeneration in patients undergoing anterior cervical discectomy and fusion based on pre-operative MRI findings. J Clin Neurosci 2014;21: 82-85.

14. Rihn JA, Lawrence J, Gates C, Harris E, Hilibrand AS. Adjacent segment disease after cervical spine fusion. Instr Course Lect 2009; 58: 747-756.

15. Sheth JH, Patankar AP, Shah R. Anterior cervical microdiscectomy: is bone grafting and in-situ fusion with instrumentation required? Br J Neurosurg 2012; 26: $12-15$.

16. Nandoe Tewarie RD, Bartels RH, Peul WC. Long-term outcome after anterior cervical discectomy without fusion. Eur Spine J 2007; 16: 1411-1416.

17. Botelho RV, Dos Santos Buscariolli Y, de Barros Vasconcelos Fernandes Serra MV, Bellini MN, Bernardo WM. The choice of the best surgery after single level anterior cervical spine discectomy: A systematic review. Open Orthop J 2012; 6: 121-128.

18. Kim JH, Park JY, Yi S, Kim KH, Kuh SU, Chin DK, Kim KS, Cho YE. Anterior cervical discectomy and fusion alters whole-spine sagittal.

19. Hankinson H, Wilson C. Use of the operating microscope in anterior cervical discectomy without fusion. J Neurosurg 1975; 43 : 452-56.

20. Cormack BMMc, Veisten PR. Cervical spondylosis. An update. West J Med 1996; 165(1-2) : 43-51.

21. Donaldson JW, Nelson PB. Anterior cervical discectomy without interbody fusion. Surg Neurol 2002; $57: 219-25$. 